\title{
On the Optimal Placement of Distributed Storage Systems for Voltage Control in Active Distribution Networks
}

\author{
M. Nick, Student Member, IEEE, M. Hohmann, R. Cherkaoui, Senior Member, IEEE \\ and M. Paolone, Senior Member, IEEE
}

\begin{abstract}
Within the context of ancillary services provided to active distribution networks by distributed storage systems (DSSs), the paper focuses on the problem of optimally locating DSSs in order to maximize their support to the network voltage control. In particular, the paper proposes an optimal planning procedure that accounts specifically for the minimization of the network voltage deviations based on the formulation of a mixedinteger linear programing problem. In view of the large size of the problem, its solution relies on the so-called benders decomposition technic. The paper discusses the performance and the sensitivities of the proposed method as function of different scenarios accounting for various loads and distributed generation (DG) profiles as well as for different number of DSSs. An application example, referring to the IEEE 13 busses test feeder, is finally included to demonstrate, and discuss, the efficiency of the proposed method.
\end{abstract}

Index Terms- Distributed energy storage systems, active distribution networks, optimal location, mixed integer non-linear problems, benders decomposition, voltage control.

\section{NOMENCLATURE}

$\left|\overline{V_{i, t}}\right|$

$\frac{\partial\left|\overline{V_{i, t}}\right|}{\partial P_{j, t}}, \frac{\partial\left|\overline{V_{i, t}}\right|}{\partial Q_{j}}$

$\frac{\partial \overline{V_{i, t}}}{\partial P_{j, t}}, \frac{\partial \overline{V_{i, t}}}{\partial Q_{j, t}}$

$\overline{V_{i, t}}, \underline{V_{i, t}}$

$V_{\text {ref }}$

$\Delta P_{j, t}$

$\Delta Q_{j, t}$

$u_{j}$
Voltage magnitude of bus $I$ at time $t$

Voltage magnitude sensitivity of bus $i$ with respect to active/reactive power injections at bus $j$, at time $t$ Partial derivatives of the voltage phasors of bus $i$ with respect to the active/reactive power at bus $j$ at time $t$

Voltage phasor of bus $i$ at time $t$, and relevant conjugate

Magnitude of the voltage reference

Variation of Active power

produced/absorbed at bus $j$ by energy

storage at time $t$

Variation of reactive power

produced/absorbed at bus $j$ by energy

storage at time $t$

Binary variable associated to the

presence of energy storage at bus $j$
M. Nick, M. Hohmann, R. Cherkaoui and M. Paolone are with the École Polytechnique Fédérale de Lausanne EPFL, Lausanne, Switzerland, (e-mail: mostafa.nick@epfl.ch, marc.hohmann@epfl.ch, rachid.cherkaoui@epfl.ch, mario.paolone@epfl.ch).

$C_{j}^{S}$
$i, j$
$t$
$n$
$A u x_{i, t}^{1}, A u x_{i, t}^{2}$
$\operatorname{Cap}_{\max , j}$

Power rating of each energy storage unit at bus $j$ (MVA)

Indexes of network nodes without the slack bus

Index of time

Index of benders iteration

Auxiliary variables for the linearization of the objective function Maximum power of the DSS at node $j$

\section{INTRODUCTION}

$T$ HE lack of direct controllability of distributed generation (DG) by distribution networks operators (DNOs) represents one of the main obstacles to maximize the penetration of traditional and renewable distributed energy sources. The difficulties associated to the near-term deployment of dedicated telecommunication infrastructures, as well as to the availability of advanced SCADA implementing active control functions, is contributing to the delay of DG direct control. One of the most promising near-term solutions that allow to postpone, in principle, the investment of the above-mentioned dedicated telecommunication infrastructures, is the possibility to indirectly control active distribution networks by means of dispersed storage systems (DSS) (e.g. [1-4]). Indeed, the availability of DSSs allows, in principle, to develop advanced control functionalities aimed at providing networks ancillary services like voltage and power flows controls. At the same time, DSSs provide typical energy balance support for the compensation of hourly, daily and weekly load/renewables absorption/generation variations (e.g. [3], [5]).

In this context, one of the main issues associated with the deployment of DSSs in active distribution networks is related to their optimal size and location. Various objective functions can be used for the relevant planning and site selections. Typical application examples refer to peak shaving [6] and energy compensation of renewable energy resources [7]. Additionally, in view of the peculiar electrical characteristics of the power distribution networks ${ }^{1}$, DSSs can provide ancillary services like voltage control support [2] and, in case of islanding operation, frequency control [4].

\footnotetext{
${ }^{1}$ A peculiar characteristic of distribution network is the non-negligible ratio between short circuit resistance and reactance. This involves, as known, the inherent coupling of voltages variations with both active and reactive power injections.
} 
In this respect, the literature on this subject has treated the following aspects: in [8] a methodology for sizing energy storage devices in microgrids context is presented. The authors used genetic algorithm to find the optimal capacities of energy storage with an objective function formulated to minimize the operation costs of microgrids. In [9] a methodology for allocating energy storage systems in a distribution network has been proposed with the aim of decreasing wind energy curtailment and minimizing annual cost of the electricity. Additionally, the subject of optimal DG location and sizing has been largely discussed with reference to various goals (e.g. [10-13]). In particular, the problem of loss minimization by optimal planning of DGs is discussed in [10]. In [11] and [12] a multi-objective problem including investment, operation, maintenance, loss, and capacity adequacy costs is used for siting and sizing of DGs. The problem of optimal DG placement in a liberized context is investigated in [13] where two different objective functions, namely, social welfare maximization and profit maximization, are taken into account.

Within the context of ancillary services provided to active distribution networks, this paper proposes an algorithm for the optimal location of DSSs for voltage control support. The proposed algorithm makes use of voltage sensitivities as a function of the nodal power injections. It aims at minimize the voltage deviations of an active distribution network over an extended time period as a function of the location of the DSSs. In particular, as it will be discussed next, the original problem consists of a mixed integer linear one. Benders decomposition [14], widely used in power system problems such as security constraint unit commitment and power system planning [1517], is adopted to decompose the original problem into a mixed-integer linear master problem and a linear sub-problem. Application examples are finally provided in order to show the features of the proposed algorithm and its benefits.

The paper is organized as follows: section 2 is devoted to the formulation of the problem with particular reference to the description of the proposed method. By making reference to a modified IEEE 13-bus test feeder [18], section 3 discusses robustness and sensitivity of the proposed method against different load and DG production scenarios as well as different maximum number of DSSs units. Section 4 concludes the paper with final remarks concerning the applicability of the proposed procedure.

\section{PROBLEM FORMULATION}

The context of the problem refers to active distribution networks characterized by the presence of non-dispatchable generation without the possibility to take advantage of demand-side management. We assume to know the phasors of phase-to-ground voltages in all the network busses at a generic time $t$. The objective of the problem is to find the best locations of DSSs that can contribute at most to minimize the voltage deviations in all the network busses. Since DSSs are assumed to be used for network control purposes, the procedure makes only reference to the DSSs siting problem and it does not considers the problems of sizing of DSSs reservoir and related investment costs. It is assumed that the maximum amount of total DSSs power is given. Additionally, it is assumed that the various DSSs locations have equal installation and operation costs.

In view of the above, the objective function could be formulated as follows:

$$
O b j=\sum_{t} \sum_{i}\left(\left|V_{r e f}-\right| \overline{V_{i, t}}||\right)
$$

Where the sum of the differences between the reference voltage magnitude and the network buses ones are minimized over a given period of time ${ }^{2}$. At bus $i$ the voltage could be expressed as the initial voltage of it plus a variation provided by the DSSs:

$$
\left|\overline{V_{i, t}}\right|=\left|\overline{V_{i, t}^{\mathrm{int}}}\right|+\Delta\left|\overline{V_{i, t}}\right| .
$$

In order to link the variations of the bus voltages to the DSSs power injections, the proposed procedure uses the voltage sensitivity coefficients. As known, each coefficient linearizes the dependency between the $i$-th bus voltage magnitude variation and the $j$-th bus injected active or reactive power variation. The typical procedure for the computation of these sensitivities is the use of an updated Jacobian matrix derived from the load flow problem (e.g. [19-21]). An analytical expression of these coefficients, that does not use the load-flow Jacobian matrix, has been recently proposed in [22]. In such an approach they are expressed as follows:

$$
\begin{aligned}
& \frac{\partial\left|\bar{V}_{i, t}\right|}{\partial P_{j, t}}=\frac{1}{\left|\bar{V}_{i, t}\right|} \operatorname{Re}\left(\underline{V_{i, t}} \cdot \frac{\partial \bar{V}_{i, t}}{\partial P_{j, t}}\right) \\
& \frac{\partial\left|\overline{V_{i, t}}\right|}{\partial Q_{j, t}}=\frac{1}{\left|\overline{V_{i, t}}\right|} \operatorname{Re}\left(\underline{V_{i, t}} \cdot \frac{\partial \overline{V_{i, t}}}{\partial Q_{j, t}}\right)
\end{aligned}
$$

It is therefore possible to compute the total variation of the voltage at bus $i$ as due to the DSS power absorption/injection at bus $j$ :

$$
\Delta\left|\overline{V_{i, t}}\right|=\sum_{j}\left(\frac{\partial\left|\overline{V_{i, t}}\right|}{\partial P_{j, t}} \Delta P_{j, t}+\frac{\partial\left|\overline{V_{i, t}}\right|}{\partial Q_{j, t}} \Delta Q_{j, t}\right)
$$

It is important to note that in (5) the inputs of the problem are the sensitivities that are function of the loads/injections in the network. It is clear that in (5) only nodes with connected DSS can inject/absorb power. The locations of the various DSSs are the unknowns of the problem and the objective is to find their optimal locations in order to obtain the minimum amount of the overall network voltage deviations within a specific time horizon. In order to account the presence or not of a DSS to a specific node, binary variables $u_{j}$ are introduced in the constraints of the problem as listed below.

$$
\begin{gathered}
\sqrt{\left(\left(\Delta P_{j, t}\right)^{2}+\left(\Delta Q_{j, t}\right)^{2}\right)} \leq C_{j}^{S} u_{j} \\
\sum_{j} u_{j} \leq \max \text { number of nodes } \\
\sum_{j} C_{j}^{S}=\text { Total Capacity }
\end{gathered}
$$

\footnotetext{
${ }^{2}$ Note that the slack-bus voltage is not considered in the objective function.
} 


$$
\begin{gathered}
C_{j}^{S} \geq u_{j} \times \text { Min Storage power rate } \\
C_{j}^{S} \leq \text { Max Storage power rate }
\end{gathered}
$$

Equation (6) refers to constraint associated to the apparent power rating of the DSS unit connected at node $j$. Equation (7), and (8) provides constraints to the total number and total power rating of DSSs. The total power of DSS that can be installed at each node is limited by a minimum and maximum power rating (constraints (9), and (10)).

\section{A. Solution methodology}

The above-formulated optimal problem is certainly a largescale mixed integer non-linear one. As known, the solution of large-scale optimal problems can be obtained by using, in general, heuristic and non-heuristic methods. In this paper we have chosen decomposition methods to break down this problem into smaller ones. In particular, the so-called benders decomposition procedure is used here to decompose the problem into a master and a sub-problem. As the objective function and the constraint (6) are non-linear, they are suitably linearized, as explained in the following, to formulate the problem in a simpler way.

The decomposition of the problem has been implemented as follows. A master problem is defined (as a mixed integer linear one) in order to determine the location of the DSSs. Then, the resulting values are imposed to a sub-problem. The solution of the sub-problem allows defining a set of dual values indicating the change in the objective function resulting from marginal variation in the DSS capacities. These are returned to the master problem as a feedback in order to setup an iterative procedure. Actually, benders cut is generated from these dual values governing the determination of the master problem solution. This process is iterated between the master problem and the sub-problem until it converges to the optimal solution. The stopping criterion of the iterative procedure is set when the variation of the objective function is negligible in comparison to the previous iteration.

The detail about the proof and the criteria for the convergence of the benders decomposition can be found in [14].

\section{1) Master problem}

The initial master problem determines the location of DSSs regardless of the sub-problem. Afterwards, at each iteration, a cut from the sub-problem is added to the master problem that is formulated as follows:

$$
\begin{aligned}
& \operatorname{Min} Z \\
& \quad Z \geq O \operatorname{Obj}^{n}+\sum_{t} \sum_{j}\left(\text { dual }_{t, j}^{S} \times\left(C_{j}^{S}-C_{j, n}^{S}\right)\right)
\end{aligned}
$$

Where $O b j^{n}$ and $C_{j, n}^{S}$ are respectively the values of the objective function and DSSs apparent power in correspondence of the previous iteration $n$. The values $d u a l_{t, i}^{S}$ are dual multipliers of the power rating constraint for all buses. The constraints of the master problem are equations (7), (8), (9), and (10).

\section{2) Sub-problem}

The sub-problem objective is to minimize the voltage deviations using the available DSS units. The objective function is the same as (1) and the constraints are (2), (5), and (6).

Since the objective function (1) is not linear, two auxiliary variables have been defined to linearize it. Thus, the subproblem is reformulated in terms of a new objective function $V_{D e v}$ given below:

$$
\begin{aligned}
& \text { Min } V_{D e v} \\
& V_{D e v} \geq \sum_{t} \sum_{i}\left(A u x_{i, t}^{1}+A u x_{i, t}^{2}\right)
\end{aligned}
$$

The following constraints are also added to the problem to ensure that the new formulation is equivalent to the original one.

$$
\begin{aligned}
A u x_{i, t}^{1}-A u x_{i, t}^{2} & =V_{r e f}-\left|\overline{V_{i, t}}\right| \\
A u x_{i, t}^{1} & \geq 0 \\
A u x_{i, t}^{2} & \geq 0
\end{aligned}
$$

Concerning the constraints of active-reactive powers injected/absorbed by DSSs they are represented as described next.

As known, DSS units are typically DC sources, therefore, they are interfaced with the grid by means of power electronic sources. These elements can be controlled to operate in all four quadrants delivering/absorbing the real/reactive power [23] and limited up to their rated current. As a consequence, the capability curve of DSS units can be represented, in the PQ, plane as shown in Fig. 1. As this constraints are also non linear, they are linearized, as shown by Fig. 1, by merging 12 linear boundaries approximating the original curve.

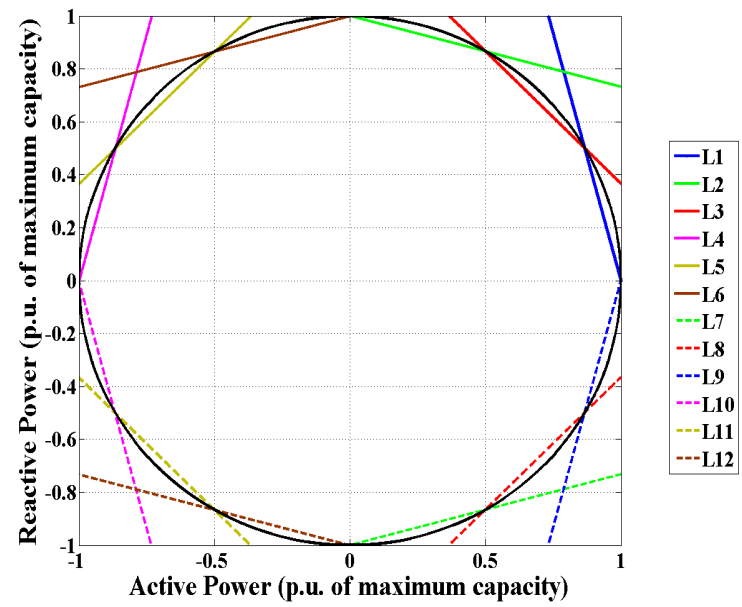

Fig. 1. Linearization of the DSS capability curve power constraint.

With reference to L1-L12 boundaries of Fig. 1, we can express the constraint (6) as follows:

$$
\begin{gathered}
\operatorname{Cap}_{\max , j}=C_{j}^{S} \\
-\operatorname{Cap}_{\max , j} \leq \Delta P_{j, t} \leq \operatorname{Cap}_{\max , j} \\
\Delta Q_{j, t} \leq \frac{1}{(\sqrt{3}-2)}\left(\Delta P_{j, t}-\operatorname{Cap}_{\max }\right) \quad L 1
\end{gathered}
$$




$$
\begin{gathered}
\Delta Q_{j, t} \leq L 2, L 3, L 4, L 5, L 6 \\
\Delta Q_{j, t} \geq L 7, L 8, L 9, L 10, L 11, L 12
\end{gathered}
$$

Where $\operatorname{Cap}_{\max , j}$ refers to the maximum power of the DSS bounded by constraint (6) given by the master problem. The dual multipliers of the constraint (16) are used to form the appropriate cut for the master problem. They show the incremental change in the objective function value while taking into account the increase of one DSS power. By adding the benders cut to the master problem, this last one is solved using the new constraints defined by (11). Fig. 2 summarizes the whole flowchart of the proposed procedure.

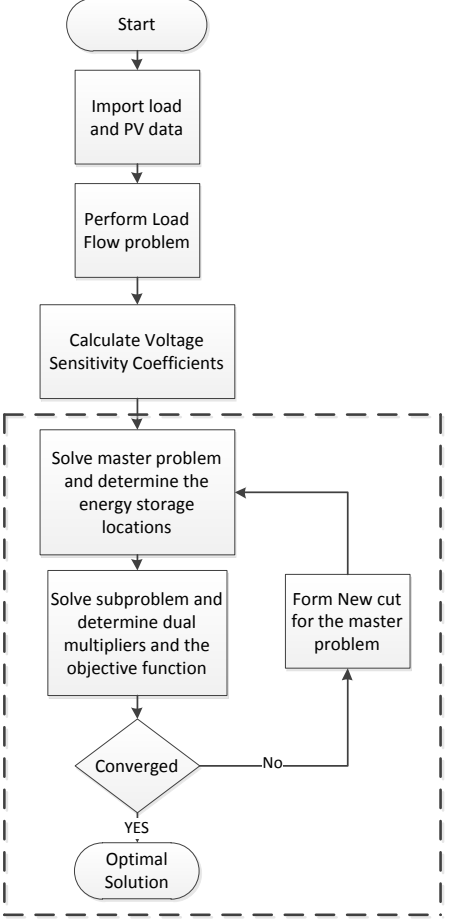

Fig. 2 Flowchart of the proposed methodology

The above/described method is implemented within MATLAB/GAMS interface. The CPLEX solver is used to find the solution of both master and sub-problem. It is worth noting that for the solution of problems with integer variables, CPLEX uses a branch and cut algorithm which solves a series of linear program sub-problems. The linear problems are solved by CPLEX's state of the art dual simplex algorithm [24].

\section{SimUlation AND RESUltS}

This section of the paper presents the use of the proposed method with reference to the IEEE 13 nodes test feeder. The schematic of this network is shown in Fig. 3. It is supposed to have non-dispatchable DG units composed by photovoltaic panels (PVs). Concerning the representation of the network loads, they are considered as voltage independent PQ absorption. The time series related to active load absorption make reference to experimentally data. The power factor of loads is considered constant during the whole time series.

The power injections of PVs have been represented by voltage-independent active power injections with null reactive component. It is assumed that the non-dispatchable PV injections are in correspondence of nodes \#646, \#684, \#675, and \#633 with a maximum rated power of $400 \mathrm{~kW}$ each. The relevant time series make reference to real measured solar irradiation in the central region of Europe. The total load and PV generation curves are shown in the Fig. 4 with reference to a one-year time horizon.

Concerning the assumptions related to: (i) maximum number of nodes where DSSs could be installed and (ii) total DSS power, they have been assumed equal to 5 and 2 MVA respectively.

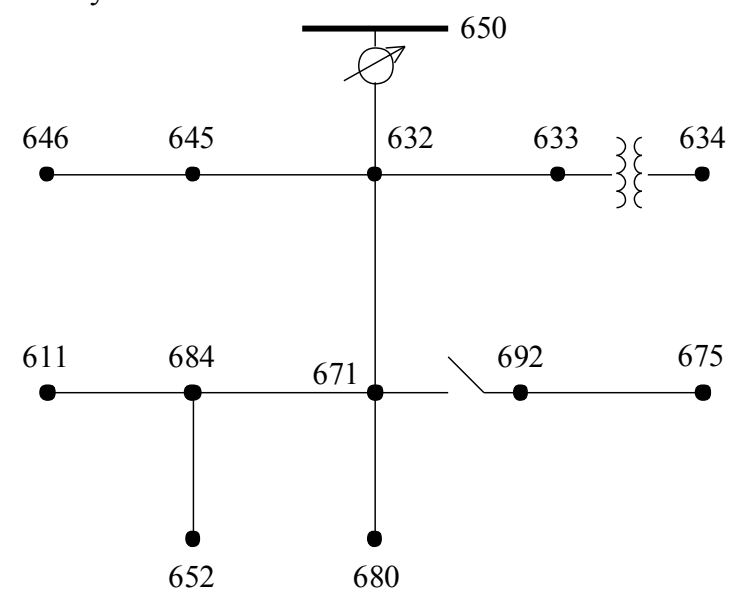

Fig. 3. Topology of the IEEE 13 node test feeder. Adapted from [18].

By making reference to a simulation window of 364 days discretized in 15 minutes time-intervals, i.e. 96 time intervals per day (see Fig. 4), Table I shows the results related to the optimal allocated DSSs capacities at each node of the network.
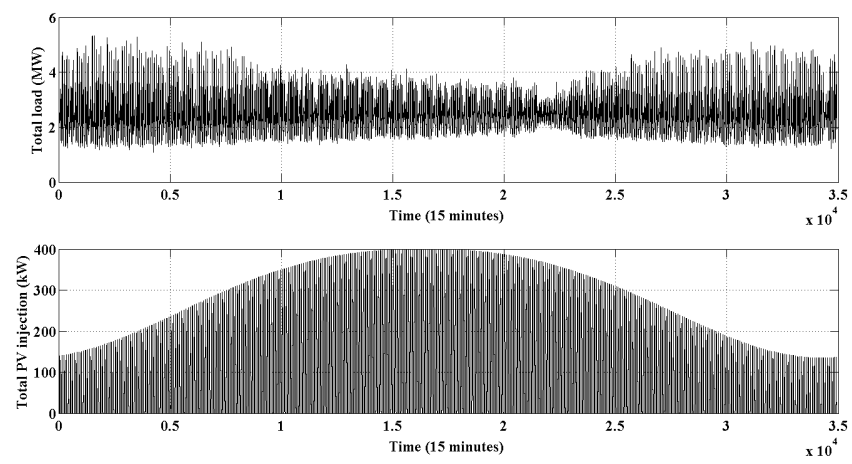

Fig. 4. Total load absorption and PV injections within a time window of one year.

TABLE I

DSSs power in optimal solution

\begin{tabular}{llllll}
\hline Node number & $\# 671$ & $\# 684$ & $\# 633$ & $\# 645$ & $\# 675$ \\
DSSs power (kVA) & 631 & 290 & 365 & 250 & 464 \\
\hline
\end{tabular}

The value of the objective function, that represents the total voltage deviation for all the considered time intervals and for all the nodes, is shown in Fig. 5. As it can be seen, the procedure allows to clearly identifying a minimum of the objective function.

The distribution density of the voltages corresponding to all network nodes for the whole simulated period is shown in Fig. 
6. In particular, Fig. 6a) refers to the case without DSSs whilst Fig. 6b) refers to the case in which DSSs are installed in agreement to the optimal location reported in Table I. As it can be observed, this last case results into voltages densities closed the value of 1 p.u..

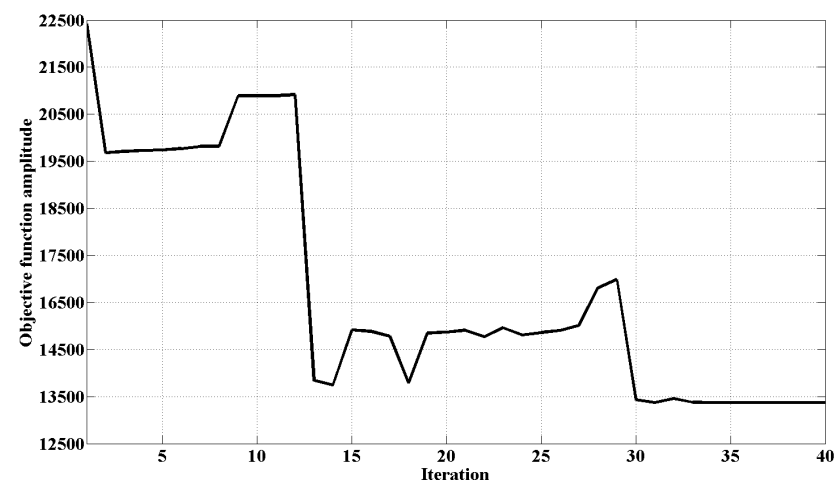

Fig. 5. Total voltage deviation in each iteration (i.e. trend of the objective function as a function of the iterations).
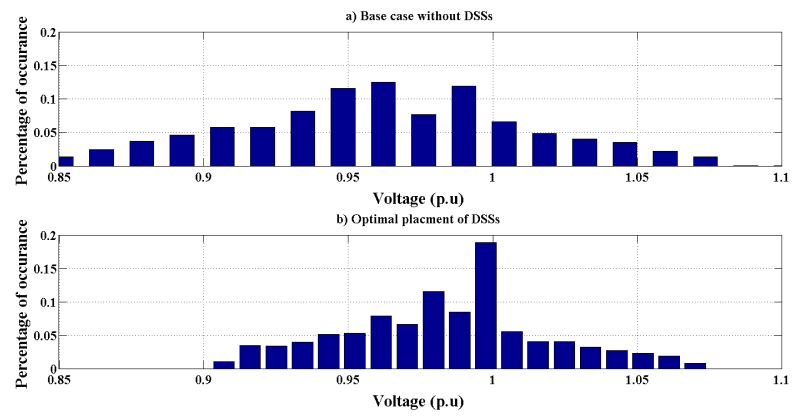

Fig. 6 Distribution of voltages in the network nodes and in all time periods

To further support the effectiveness of the proposed method, we here propose a sensitivity analysis of the optimal location solution against the variation of the maximum number of nodes where DSSs can be installed. In particular, this number is varied from 0 to 5 by keeping constant the total DSSs power (i.e. $2 \mathrm{MVA}$ ). In order to perform this analysis, we have selected two generic weeks of the entire time period (for instance, the first week of the year and a week in the middle of the year). For each time period of the selected week, we have computed the norm of the network voltages. The cumulative distribution functions of these norms are shown in Fig. 7 for the six analyzed cases. The results of this figure show that, with a fixed amount of total DSSs power, when the number of possible nodes where DSSs could be installed increases the overall network voltage could be better controlled. Additionally, all cases referring to the presence of DSSs shows better voltage controllability compare to the case without DSS.

In order to verify the robustness of the proposed method against different scenarios (i.e. determine whether the optimal location of DSS remains essentially unchanged with different boundary conditions), we here compare the optimal solutions of the problem obtained by using two sets of power absorptions/injections. The first data set refers to a time window corresponding to the first six months of the year whilst the second one makes use of the subsequent six months. The relevant results are shown in Table II. Although the first and second half of the considered time period (i.e. one year) are not completely symmetrical (see Fig. 4.) the proposed method results into optimal locations which are essentially identical (with the exception of nodes \#633 and \#634). Even the allocated power of DSS units shows a consistency between the two obtained optimal solutions. These results allow to conclude that, if the considered time window is representative of the majority of the changes in power injections and absorptions, the obtained optimal location remains unchanged.

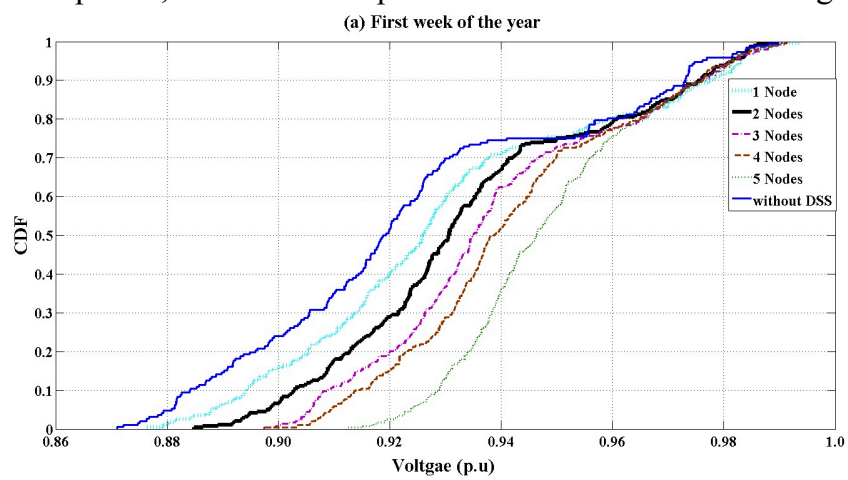

(b) 25 th week of the year

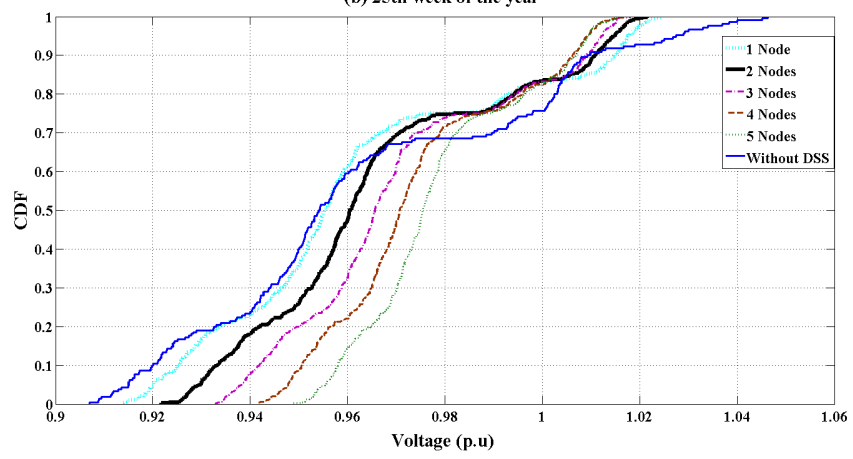

Fig. 7. CDF of voltages norm for different number of DSSs location.

TABLE II

Optimal solution for the first and second half of the year

\begin{tabular}{|c|c|c|c|c|c|c|}
\hline Node number & \#671 & $\# 684$ & \#633 & $\# 645$ & $\# 675$ & $\# 634$ \\
\hline $\begin{array}{l}\text { DSS power corresponding } \\
\text { to the first scenario (first } \\
\text { half of the year) (kVA) }\end{array}$ & 605 & 260 & 0 & 208 & 510 & 417 \\
\hline $\begin{array}{l}\text { DSS power corresponding } \\
\text { to the second scenario } \\
\text { (second half of the year) } \\
\text { (kVA) }\end{array}$ & 640 & 310 & 408 & 230 & 412 & 0 \\
\hline
\end{tabular}

\section{CONCLUSIONS}

The paper has focused on the description of a planning procedure applied to the optimal siting of DSSs with the purpose of maximizing their impact in terms of voltage support to active distribution networks. The paper has shown the formulation of the optimal problem and has also proposed a feasible procedure for its solution based on the use of the socalled benders decomposition methodology.

The use of the proposed procedure for the optimal siting of an a-priori fixed total power of DSSs in the IEEE 13 bus test feeder, has shown its capability to find optimal DSS locations accounting: (i) a large time horizon, (ii) a realistic distribution 
network configuration and (iii) realistic load and renewables power absorption/production profiles.

The sensitivity analysis discussed in the paper has allowed to verify the performances and the robustness of the proposed method against the variation of both load/injection scenarios and number of maximum DSS units to be installed in the network. These characteristics represent major advantages of the proposed approach as it could take into account scenarios referring to seasonal changes of both loads and stochastic DG production.

\section{REFERENCES}

[1] A. Oudalov, C. Yuen, M. Holmberg, "Energy storage is a key smart grid element", Proc. of the Cigré 2011 Bologna Symposium The Electric Power System of the Future, Bologna, Italy, Sept. 13-15, 2011.

[2] M. Zillmann, Y. Ruifeng, T.K. Saha, "Regulation of distribution network voltage using dispersed battery storage systems: A case study of a rural network", Proc. of the 2011 IEEE PES General Meeting, Detroit, USA, July 24-28, 2011.

[3] A. Gabash and P. Li, "Active-reactive optimal power flow in distribution networks with embedded generation and battery storage", IEEE Trans. on Power Systems, to be published.

[4] P. Mercier, R. Cherkaoui, A. Oudalov, "Optimizing a battery energy storage system for frequency control application in an isolated power system," IEEE Trans. on Power Systems, vol. 24, no: 3, pp: 1469 1477, Aug. 2009.

[5] K.C. Divya, J. Østergaard "Battery energystorage technology for power systems-An overview," Electric Power Systems Research, vol. 79, no. 4, p. 511-520, Apr. 2009.

[6] A. Oudalov, R. Cherkaoui, A. Beguin, "Sizing and optimal operation of battery energy storage system for peak shaving application", Prof. of the 2007 IEEE Lausanne Powertech, Lausanne-Switzerland, July 1-5, 2007

[7] J. P. Barton, and D. G. Infield, "Energy storage and its use with intermittent renewable energy," IEEE Trans. on Energy Conv., vol. 19, no. 2, pp. 441-448, Jun. 2004.

[8] C. Chen, S. Duan, T. Cai, B. Liu, and G. Hu "Optimal allocation and economic analysis of energy storage system in microgrids," IEEE Trans. on Power Electronics, vol. 26, no. 10, pp. 2662-2773, October 2011.

[9] Y. M. A. E. F. El-Saadany, "Optimal allocation of ESS in distribution systems with a high penetration of wind energy," IEEE Trans. on Power Systems, vol. 25, no. 4, pp. 1815-1822, November 2010.

[10] Y. M. Atwa, E. F. El-Saadany "Analytical approaches for optimal placement of distributed generation sources in power systems," IEEE Trans. on Power Systems, vol. 19, no. 4, pp. 2068-2076, November 2004.

[11] G. Celli, E. Ghiani, S. Mocci, F. Pilo "A multi-objective evolutionary algorithm for the sizing and siting of distributed generation," IEEE Trans. on Power Systems, vol. 20, no. 2, pp. 750-757, May 2005.

[12] Z. Liu, F. Wen, G. Ledwich, "Optimal siting and sizing of distributed generators in distribution systems considering uncertainties," IEEE Trans. on Power Delivery, vol. 26, no. 4, pp. 2541-2551, October 2011.

[13] D. Gautam, N. Mithulananthan "Optimal DG placement in deregulated electricity market," Electric Power Systems Research, vol. 77, no. 12 p. 1627-1636, 2007.

[14] A. Conejo, E. Castillo, R. Minguez, R. Garcia-Bertrand Decomposition techniques in mathematical programming, Springer, 2006.

[15] T. Niknam, A. Khodaei, F. Fallahi, "A new decomposition approach for the thermal unit commitment problem", Applied Energy, Vol. 68, no 9, pp. 1667-1674, 2009

[16] S. Binato, F. Pereira, S. Granville "A new benders decomposition approach to solve power transmission network design problems," IEEE Trans. on Power Systems, vol. 16, no. 2, pp. 235-240, may 2001.

[17] J. Roh, M. Shahidehpour, Y. Fu, "Security-constrained resource planning in electricity markets," IEEE Trans. on Power System, vol. 22, no. 2, pp. 812-820, May 2007.

[18] IEEE Distribution Planning Working Group, "Radial distribution test feeders," IEEE Trans. Power Syst., vol. 6, no. 3, pp. 975-985, Aug. 1991

[19] J. Peschon, D. Piercy, W. Tinney, and O. Tveit, "Sensitivity in power systems," IEEE Transactions on Power Apparatus and Systems, no. 8, pp. 1687-1696, 1968.

[20] D. Shirmohammadi, H. Hong, A. Semlyen, and G. Luo, "A compensation-based power flow method for weakly meshed distribution and transmission networks," IEEE Trans. on Power Systems, vol. 3, no. 2, pp. 753-762, 1988.

[21] A. Wood and B. Wollenberg, Power generation, operation, and control. Wiley New York, 1996, vol. 2.

[22] K. Christakou, J.-Y. Le Boudec, M. Paolone, D.-C Tomozei, "Efficient computation of sensitivity coefficients of node voltages and Line currents in unbalanced electrical networks", Cornell University Library, ref. 1203

[23] E. Muljadi, C.P. Butterfield, R. Yinger, and H. Romanowitz "Energy storage and reactive power compensator in a large wind farm" 42nd AIAA Aerospace Sciences Meeting and Exhibit Reno, Nevada January 5-8, 2004.

[24] GAMS - The Solver Manuals, available at "http://www.gams.com/dd/docs/solvers/allsolvers.pdf.

\section{BIOGRAPHIES}

Mostafa Nick was born in IRAN in 1986. He received the Master degree in electrical engineering from Amirkabir University of Technology (Tehran Polytechnic), in 2011. Currently he is pursuing his $\mathrm{PhD}$ degree in the Distributed Electrical System laboratory (DESL) of the École Polytechnique Fédérale de Lausanne (EPFL), Lausanne, Switzerland. His current research interests include planning problems of active distribution networks, distributed energy storage modeling and relevant applications for real-time operation of electrical distribution grids.

Marc Hohmann was born in 1989. He received a Bachelor degree in electrical engineering from the École Polytechnique Fédérale de Lausanne (EPFL), Lausanne, Switzerland where he is currently pursuing a Master degree in electrical engineering His research interests are in the areas of power systems, power electronics and controls.

Mario Paolone (M'07-SM'10) was born in Campobasso, Italy, in 1973. He received the M.Sc. degree (with honors) in electrical engineering and the $\mathrm{Ph} . \mathrm{D}$. degree from the University of Bologna, Italy, in 1998 and 2002, respectively.

In 2005, he was appointed Researcher in Electric Power Systems at the University of Bologna where he was with the Power Systems Laboratory until 2011. In 2010, he received Associate Professor eligibility from the Politecnico di Milano, Italy. Currently, he is Associate Professor at the Swiss Federal Institute of Technology, Lausanne, Switzerland, where he accepted the EOS Holding Chair of Distributed Electrical Systems Laboratory. He is secretary and member of several IEEE and Cigré Working Groups. He was cochairperson of the technical committee of the ninth edition of the International Conference of Power Systems Transients. His research interests are in the area of smart grids, with particular reference to real-time monitoring and operation, power system protections, power systems dynamics, and power system transients with particular reference to LEMP-interaction with electrical networks.

Rachid Cherkaoui received both the M.Sc. and Ph.D. degrees in electrical engineering in 1983 and 1992, respectively, from the Electrical \& Electronic Institute at the École Polytechnique Fédérale de Lausanne (EPFL), Switzerland. He is now a Senior Research Engineer at EPFL, LRE. His research interests are in electricity market deregulation, distributed generation and storage, and power system vulnerability mitigation. He is member of technical program committees of various conferences, member of CIGRE TF's and WG's, and IEEE chapter officer since 2005. He is author and coauthor of more than 50 scientific publications. 